\title{
Mesenchymal Stem Cell Therapy for COVID-19: Present or Future
}

\author{
Ali Golchin ${ }^{1}$ (D) Ehsan Seyedjafari ${ }^{2} \cdot$ Abdolreza Ardeshirylajimi $^{3,4}$
}

Published online: 13 April 2020

(C) Springer Science+Business Media, LLC, part of Springer Nature 2020

\begin{abstract}
"COVID-19" is the word that certainly isn't forgotten by everybody who lives in the first half of the twenty-first century. COVID19, as a pandemic, has led many researchers from different biomedical fields to find solutions or treatments to manage the pandemic. However, no standard treatment for this disease has been discovered to date. Probably, preventing the severe acute respiratory infection form of COVID-19 as the most dangerous phase of this disease can be helpful for the treatment and reduction of the death rate. In this regard, mesenchymal stem cells (MSCs)-based immunomodulation treatment has been proposed as a suitable therapeutic approach and several clinical trials have begun. Recently, MSCs according to their immunomodulatory and regenerative properties attract attention in clinical trials. After the intravenous transplantation of MSCs, a significant population of cells accumulates in the lung, which they alongside immunomodulatory effect could protect alveolar epithelial cells, reclaim the pulmonary microenvironment, prevent pulmonary fibrosis, and cure lung dysfunction. Given the uncertainties in this area, we reviewed reported clinical trials and hypotheses to provide useful information to researchers and those interested in stem cell therapy. In this study, we considered this new approach to improve patient's immunological responses to COVID-19 using MSCs and discussed the aspects of this proposed treatment. However, currently, there are no approved MSC-based approaches for the prevention and/or treatment of COVID-19 patients but clinical trials ongoing.
\end{abstract}

Keywords COVID-19 $\cdot$ Coronavirus $\cdot$ Mesenchymal stem cell $\cdot$ Stem cell therapy $\cdot$ Immunomodulatory $\cdot$ Clinical trials

\section{Introduction}

At the 2019 year's end, numerous cases of severe respiratory infections were reported in Wuhan, China, and were initially thought to be a seasonal flu disease, given that some patients had a history of attending or working in the wholesale market for fish and seafood. The market was immediately shut down on January 1, and environmental sanitation and sanitation

Ali Golchin

agolchin.vet10@yahoo.com

$\triangle$ Abdolreza Ardeshirylajimi

r.ardeshiry.62@gmail.com

1 Department of Clinical Biochemistry and Applied Cell Science, School of Medicine, Urmia University of Medical Sciences, Urmia, Iran

2 Department of Biotechnology, College of Science, University of Tehran, Tehran, Iran

3 Urogenital Stem Cell Research Center, Shahid Beheshti University of Medical Sciences, Tehran, Iran

4 SinaCell Research and Product Center, Tehran, Iran were fully implemented. A few days later, after rejecting the diagnosis of seasonal influenza, avian influenza, adenovirus, coronavirus, SARS, coronavirus, and other pathogens, on Jan. 1, the virus was declared a causative agent of the disease in four of the nine hospitalized patients: A new coronavirus that has a 5\% genetic association with SARS and is a subset of Sarbecovirus [1]. Currently, the virus has been briefly named SARS-CoV-2 virus for further information and COVID-19, the name was given by the World Health Organization (WHO) to the SARS-CoV-2 virus-associated disease.

This disease has resulted in that clinicians and researchers from different branches of biomedicine were mobilized to find a solution or treatment for the management of this pandemic. According to a recent announcement of the International Society for Stem Cell Research (ISSCR), currently, there are no approved stem cell-based approaches for the prevention and treatment of COVID-19 infection. However, recently, mesenchymal stem cells (MSCs) have introduced one of the therapeutic approaches for using in the treatment of COVID19 [2]. As we know, MSCs opposes viral infection due to the presence of specific cytokines improved qualities. These features are present in MSCs in the intrinsic niche before their separation process happens. Therefore, MSCs can be expected 
to survive even if they are transplanted into a patient with a confirmed COVID-19. Due to there is disagreement in MSCs therapy to treat COVID-19, we reviewed reported clinical trials and news to present helpful information to researchers and enthusiasts of the stem cell-based therapy field. In this study, we considered this proposed approach to improve patient's immunological responses to COVID-19 using MSCs and discussed the aspects of this therapeutic approach.

\section{SARS-CoV-2 and COVID-19}

"COVID-19" the word that certainly it isn't forgotten by everybody who lived in the first half of the twenty-first century. Coronavirus disease 2019 which known as COVID-19 is the result of one coronavirus infection in the name of SARS-CoV2. Coronaviruses $(\mathrm{CoV})$ are a large family of viruses that some of them are more known such as Middle East Respiratory Syndrome (MERS-CoV) and Severe Acute Respiratory Syndrome (SARS-CoV), but some of them are not more known like Severe acute respiratory syndrome coronavirus 2 (SARS-CoV-2). SARS-CoV-2 that previously known by the 2019 novel coronavirus (2019-nCoV), is a new strain of coronavirus that hasn't been identified in humans up to late December of 2019. However, there are reports that demonstrate the SARS-CoV-2 virus originated from bats and then moving into camels, but its exact dynamics are currently unknown. Moreover, much of the pathogenesis information regards to SARS-CoV-2 is not fully known.

SARS-CoV-2 is from the Nidovirales order, a member of the genus $\beta$-coronavirus ( $\beta-\mathrm{CoV}$ ) [3]. $\beta-\mathrm{CoV}$ includes five subgenus including in embecovirus (including $\mathrm{HCoV}-\mathrm{OC} 43$ and $\mathrm{HCoV}-\mathrm{HKU} 1$ ) [4], nobecovirus (including BtCoVHKU9) [4], hibecovirus (including Bat Hp-betacoronavirus Zhejiang2013) [4], sarbecovirus (including SARSr-CoV and its strains such as SARS-CoV, SARS-CoV-2, and Bat SLCoV-WIV1), merbecovirus (including Middle East respiratory syndrome (MERS)-CoV, BtCoV-HKU4, and BtCoVHKU5) (https://talk.ictvonline.org/taxonomy/) [4, 5].

SARS-CoV-2 is enveloped, positive-sense, single-stranded RNA virus (with nucleocapsid) by $79.6 \%$ sequence identity the same to SARS-CoV [6] that that known as the largest discovered RNA viruses by approximately $30 \mathrm{~kb}$ in length genome structure. The genome sequence of SARS-CoV-2 has been submitted to GISAID (https://www.gisaid.org/; accession number: EPI_ISL_402124). It was first isolated using human airway epithelial cells [7] but can be isolated from the bronchoalveolar lavage fluid from a COVID-19 patient $[6,7]$. Generally, both of the SARS-CoV and the SARSCoV-2 are isolated and grew readily in Vero cells $[7,8]$ (a lineage of cells that was isolated from kidney epithelial cells of an African green monkey [9]). Also, this virus as SARS-
$\mathrm{CoV}$ enters its host cell by binding to the angiotensinconverting enzyme 2 (ACE2) receptor [10, 11].

On March 11, 2020, the World Health Organization (WHO) characterized the spread of COVID-19 as a pandemic that it has caused unreasonable fear and led to unnecessary suffering and death [12]. To date (29 March 2020), according to the Worldomete site (https://www.worldometers.info/ coronavirus/) report more than 199 countries and territories around the world have been affected, with major outbreaks respectively in the USA, Italy, central China, Spain, Germany, and Iran. The mortality rate of COVID-19 has been reported from $0.7 \%$ [13] to $15 \cdot 2 \%$ [14] according to different studies in diverse territories and countries. As well, its maximum incubation period has been assumed 2 weeks [15] to 8 weeks $[14,16]$. COVID-19 has resulted in that many researchers from different branches of biomedicine were attracted to find a solution or treatment for the management of this pandemic. However, to date haven't been discovered the standard cure for this disease.

Several studies have shown that the first stage of the pathogenesis of this type of virus is the identification of angiotensin-converting enzyme-2 (ACE2) receptor by its spike protein [17]. For this reason, ACE2-positive cells are infected by this virus [17]. Another study has shown that the cellular protease TMRRSS2 is also required to allow the entry of coronavirus into host cells [18]. It is conceivable that the ACE2 receptor is widely distributed on the surface of human cells, especially alveolar type 2 (AT2) and capillary epithelium, and AT2 cells largely express TMPRSS2 [18]. On the other hand, interestingly, bone marrow, lymph nodes, thymus, spleen, and immune cells, such as T and B lymphocytes and macrophages are always negative for ACE2 (Fig. 1) [19]. These findings suggest that immunoglobulin therapy can help treat patients with the virus infection. Therefore, it should be noted that the capacity of the virus is greatly diminished by the cytokine-induced storm of the virus. The current hallmark of SARS-CoV-2 pathogenesis is the cytokine storm in the lung. Virally-triggered acute cytokine release of GSCF, IP10, MCP1, MIP1A, IL-2, IL-6, IL-7, and TNF results in pulmonary edema, dysfunction of air-exchange, acute respiratory distress syndrome (ARDS), and acute cardiac injury, and leading to death [2].

To date, there is no specific cure for Covid-19, although clinical management of these patients currently includes prevention or control of the infection and supportive care, including supplemental oxygen and mechanical ventilation support when needed. Recently, in the viral surface glycoprotein, several epitopes, including in 5 CTL epitopes, 3 sequential B cell epitopes, 5 discontinuous B cell epitopes of immune cells [20], and 13 MHC-I and 3 MHC-II antigenic epitopes [21], have been reported via immuno-informatics approach that some of these epitopes maybe had potential candidates for the development of 2019-nCoV vaccines. 


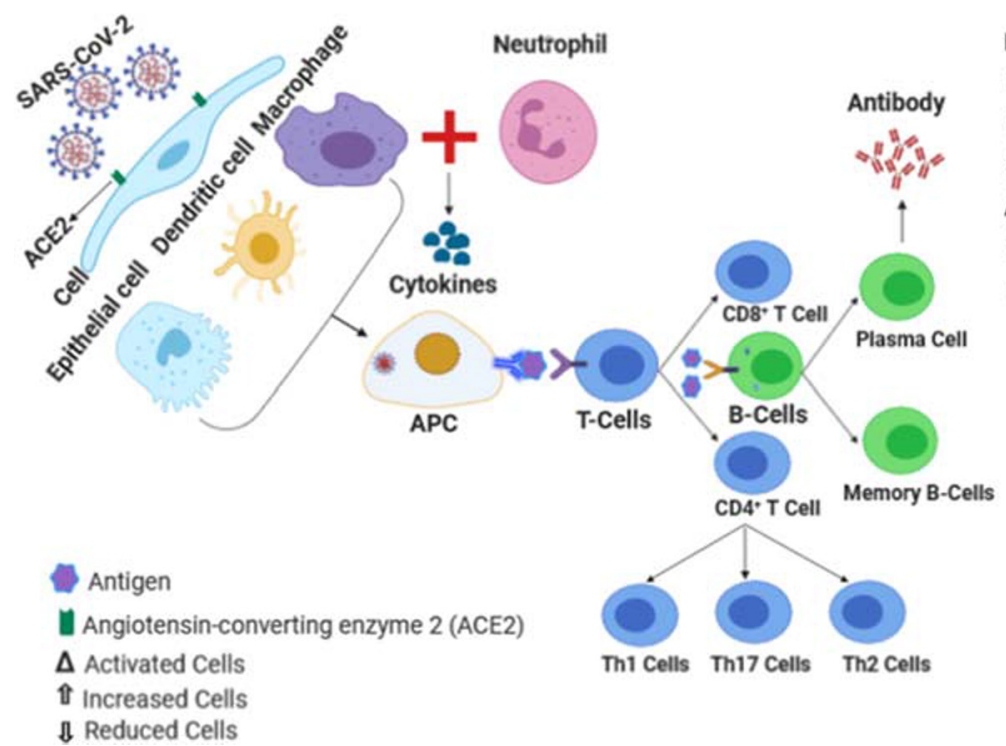

Mild-to-Moderate phase:

«Antibody-secreting cells (ASCS)

作ollicular helper T cells (TFH cells)

$\checkmark \mathrm{CD}^{*} \mathrm{~T}$ cells and $\mathrm{CD8}{ }^{*} \mathrm{~T}$ cells

$\triangle \mathrm{CDA}^{*} \mathrm{~T}$ cells and CD8* $T$ cells

^ิ|mmunoglobulin M (IgM) antibodies

$\uparrow$ Immunoglobulin G (IgG) antibodies

Acute phase:

\& lymphocytes (lymphopenia)

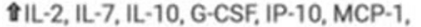

MIP-1A, and TNFa

$\Delta$ neutrophils and monocytes/macrophages

$\Delta$ Specific Th1/Th17

$\Delta$ Plasma cells

ICD $4^{*} \mathrm{~T}$ cells and $\mathrm{CD} 8^{*} \mathrm{~T}$ cells

Cytokine storm

Fig. 1 Schematic of host immune system responses during SARS-CoV-2 infection; Data obtained from [31, 32] (Figure is made with biorender: https:// biorender.com/)

A series of approved drugs for other symptoms is currently underway in clinical trials for these patients, including Chloroquine, Hydroxychloroquine, and Remdesivir worldwide. This because, safe, timely and effective supportive treatments are the inevitable principle in patients who develop severe manifestations of COVID19.

\section{Mesenchymal Stem Cell Therapy}

Currently, cell-based therapy and especially stem cell therapy has become a promising therapeutic field, in which many see opportunities to cure incurable diseases [22]. Despite the significant development of the stem cell-based therapy field, immunogenicity, limited cell source and ethical issue as the main limitations of this therapeutic approach have not been solved yet. Among these, MSCs has attracted attention due to source potential, a high proliferation rate, low invasive procedure, and free of ethical issues. There is much superiority in using MSC therapy in comparison with other treatments [23], including in I) They are easily accessible and can be isolated from various tissues such as bone marrow and adipose tissues, including in umbilical cord, dental pulp, menstrual-blood, buccal fat pad, fetal liver, etc.; II) They are multipotent stem cells; III) MSCs can easily expand to clinical volume in a suitable period of time; IV) MSCs can be stored for repetitive therapeutic usage; V) Clinical trials of MSCs so far haven't shown adverse reactions to allogeneic MSC; VI) Safety and effectiveness of MSCs have been obviously documented in several clinical trials [23].

As mentioned, Following the COVID-19, may trigger a destroying immune overreaction in the body. In COVID-19 patients, the immune system produces large amounts of inflammatory factors, causing a cytokine storm including, in an overproduction of immune cells and cytokines [24]. Here, it is the beginning of the MSC therapy idea in the treatment of COVID-19 patients. Probably, MSC therapy can prevent the storm release of cytokines by the immune system and promote endogenous repair by reparative properties of the stem cells (Fig. 2).

After intravenous injection, part of the MSC population entraps in the lung, which often in systemic infusion it is remembered as a limitation. But here these MSCs could recover the pulmonary microenvironment, protect alveolar epithelial cells, intercept pulmonary fibrosis, and cure lung dysfunction and COVID-19 pneumonia [25]. However, one of the main restrictions in this approach is the suppling source of clinical-grade MSCs and subsequently the speed of preparation for clinical usage that here stem cell banks can play an important role. Also, MSCs can be isolated from different adult tissues, including preferably bone marrow (BM), peripheral blood (PB) and adipose tissues (AT) (such as abdominal fat, infrapatellar fat pad, and buccal fat pad) and neonatal birth-associated tissues, including placenta (PL), umbilical cord (UC), Warton jelly (WJ), amniotic fluid (AF), and cord blood (CB), and then stored for future possible applications. Therefore, it seems MSCs-based therapy may possibly be an ideal candidate for clinical trials or at least the combination of treatment to treat COVID-19 patients.

\section{MSC Clinical Trials for COVID-19}

Recently, China, USA, Jordon, Iran, and several other countries have begun cell-based therapy clinical studies and some reports have been published (Table 1). Interestingly, one of the 
Fig. 2 Proposed interaction of MSCs with host immune cells and released cytocines; Data obtained from [33, 34], The figure is made with biorender (https://biorender. $\mathrm{com} /$ )

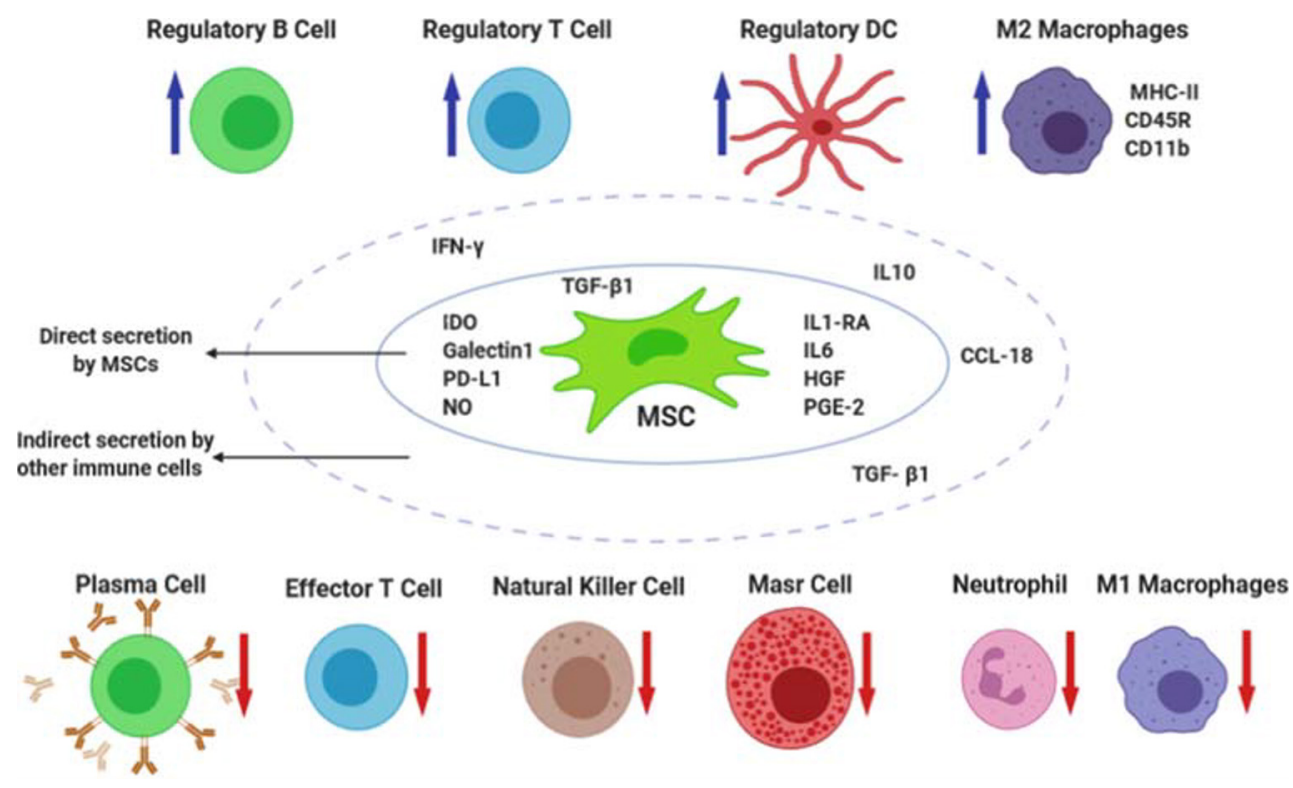

available methods to evaluate its efficacy in the maintenance or repair of damaged vital organs is the use of mesenchymal stem cell (MSCs) therapy that widely used in the treatment of type 2 diabetes, autoimmune disease, spinal cord injury, GVHD and several other diseases specially with high immunity rates have been used [23, 26-28]. MSCs, using their immunomodulatory properties and their differentiation ability, can prevent lung tissue death by counteracting the cytokine storm and regeneration and reconstruction of damaged tissues (Fig. 2). Recently, the use of these cells in the clinical treatment of $\mathrm{H} 5 \mathrm{~N} 1$ viral infections that have similar effects on the lung has also been suggested [29]. In addition, recently a case
Table 1 List of registered Cellbased clinical trials for treating COVID-19

\begin{tabular}{|c|c|c|}
\hline Clinical trial No & Cell Source & Ref \\
\hline ChiCTR2000031319 & Allogeneic Human Dental Pulp- MSC & http://www.chictr.org.cn \\
\hline ChiCTR2000031139 & Human embryonic stem cell-derived M cells (CAStem) & \\
\hline ChiCTR2000030944 & human NK cells and MSCs transplantation & \\
\hline ChiCTR2000030509 & NK Cells & \\
\hline ChiCTR2000030329 & Umbilical cord blood CIK and NK cells & \\
\hline ChiCTR2000030300 & human umbilical cord-MSCs (huc-MSCs) & \\
\hline ChiCTR2000030224 & MSCs & \\
\hline ChiCTR2000030173 & hUC-MSCs & \\
\hline ChiCTR2000031319 & Human MSCs & \\
\hline ChiCTR2000030088 & Umbilical cord Wharton's Jelly derived-MSC & \\
\hline ChiCTR2000030020 & MSCs & \\
\hline ChiCTR2000029816 & hUCB-MSCs & \\
\hline ChiCTR2000029812 & Umbilical cord blood mononuclear cells & \\
\hline ChiCTR2000029606 & human Menstrual blood-derived stem cells & \\
\hline ChiCTR2000029580 & Ruxolitinib in combination with MSCs & \\
\hline ChiCTR2000029572 & Umbilical cord blood mononuclear cells & \\
\hline ChiCTR2000029569 & Umbilical cord blood mononuclear cells & \\
\hline CTR2000030116 & hUC-MSCs & \\
\hline ChiCTR2000030484 & HU-MSCs and Exosomes & \\
\hline ChiCTR2000030866 & hUC-MSCs & \\
\hline ChiCTR2000030835 & hUC-MSCs & \\
\hline ChiCTR2000030138 & hUC-MSCs & \\
\hline NCT04313322 & Wharton's Jelly derived-MSC & https://clinicaltrials.gov \\
\hline NCT04315987 & NestCell@-MSC & \\
\hline NCT04302519 & Dental Pulp- MSCs & \\
\hline NCT04288102 & MSCs & \\
\hline NCT04273646 & UC-MSC & \\
\hline NCT04252118 & MSCs & \\
\hline NCT04299152 & MSCs & \\
\hline NCT04269525 & UC-MSCs & \\
\hline NCT04276987 & MSCs-derived exosomes & \\
\hline
\end{tabular}


study was reported in China on a female patient with an acute COVID19 syndrome that the results of laboratory tests and CT images provided extremely effective results after 21 days of treatment with umbilical cord MSCs. A recent case study of a case report of a 65-year-old female patient diagnosed in critical condition with COVID-19, then identified the exact $2019 \mathrm{nCoV}$ variant now called SARS-CoV-2 [30]. The patient had a neutrophil increase of $87 \%$ and a lymphocyte decrease of $9.8 \%$ and was treated with antiviral drugs such as lopinavir / ritonavir, IFN- $\alpha$ and oseltamivir as well as intravenous injection of moxifloxacin, Xuebijing, methylprednisolone and immunoglobulin. The patient was also subjected to noninvasive mechanical ventilation to facilitate breathing and relieve muscle fatigue due to poor oxygenation. As the vital signs worsened, the patient was treated with cord MSCs alone and with $\alpha 1$ thymosin $5 \times 10^{7}$ cells each three times. The results of the study showed that after the second injection, serum albumin, CRP, and ALT / AST gradually decreased, as well as other vital signs improved. Thereafter, the patient was removed from the ventilator and able to walk, and the number of white blood cells and neutrophils in the patient decreased to a normal level, while the number of lymphocytes increased to their normal level. Most importantly, $\mathrm{CD}^{+}{ }^{+} \mathrm{T}$ cell, $\mathrm{CD}^{+}{ }^{+} \mathrm{T}$ cell and $\mathrm{CD}^{+} \mathrm{T}$ cell numbers were significantly increased. Also, the qualitative results obtained from CT images after the second and third injections of cord stem cells showed that the pneumonia was very relieved, 2 days after the third injection the patient was discharged from the ICU ward and most of the vital signs and clinical laboratory parameters in the They were normal. The results suggested that umbilical cord mesenchymal stem cells could be an ideal treatment option alone or in combination with other immune modulators for acute COVID-19 patients [30]. In another study released recently in China and in collaboration with the United States, 7 patients with COVID19 pneumonia in Beijing YouAn Hospital from January 23 to February 16 underwent mesenchymal stem cell transplantation and clinical manifestations, changes in immune function levels [25]. Also, inflammation was assessed within 14 days after transplantation. The results showed that the clinical symptoms of all patients improved significantly 2 days after stem cell transplantation. Among the patients studied, one was very acute and two patients with milder conditions were discharged from the hospital 10 days after transplantation. Their results also showed that peripheral lymphocyte levels increased, activated cytokine-secreting immune cells, such as $\mathrm{CXCR}^{+} \mathrm{CD}^{+}{ }^{+} \mathrm{T}$ cells, $\mathrm{CXCR}^{+} \mathrm{CD}^{+} \mathrm{T}$ cells and $\mathrm{NK} \mathrm{CXCR}^{+}$cells disappeared on day 6-6. A group of $\mathrm{CD} 14^{+} \mathrm{CD} 11 \mathrm{c}^{+} \mathrm{CD} 11 \mathrm{bmid}$ regulatory DC cell populations also increased dramatically. At the same time, TNF- $\alpha$ levels were significantly decreased, whereas IL-10 was increased in patients treated with MSCs compared with patients treated with conventional therapy. In addition, gene expression profiling of mesenchymal stem cells showed that these cells are
ACE2- and TMPRSS2-, which showed that mesenchymal stem cells are free of COVID19 infection [25]. Therefore, they concluded that MSCs would be safe and effective for treating patients with COVID19 pneumonia, especially for patients with very acute conditions.

\section{Conclusion}

Immunomodulatory and anti-inflammatory properties of MSCs in the treatment of respiratory diseases were confirmed by 17 completed clinical studies, and also more than 70 trials are registered in this regard (https://clinicaltrials.gov). To date, 20 clinical trials have been registered in the Chinese clinical trial registry site (http://www.chictr.org.cn). In addition, 9 clinical trials have been registered in Clinicaltrial.gov. Umbilical cord, umbilical cord blood, Wharton's jelly, menstrual blood, dental pulp, and the company producedMSCs are the important MSC sources that will be used in these trials. However, the process of developing new therapeutic and bringing it to clinical application has important practical implications and not over for MSC therapy of COVID-19. However, the cost-effective and speed of therapeutic preparation are the capable discussed topic for MSCbased therapy for COVID-19, but certainly, the life of a human is more worthy and COVID-19 is so dangers. Therefore, the clinical use of MSCs therapy to treat COVID-19 is still some time away, but there are some promising reports to apply. Stem cell therapy and especially MSCs may possibly be is one of the most ideal therapeutics, or a combination of treatment to treat COVID-19 patients. However, scientists are trying incessantly to develop a vaccine for COVID-19, as well as therapeutics to treat this disease.

Authors' Contributions A.G. conceptualized the outline and topic of the article. All authors participated in designing the study, drafting, writing and editing the manuscript, and approving it for submission.

\section{Compliance with Ethical Standards}

Conflict of Interest We have no conflicts of interest.

\section{References}

1. Wu, F., Zhao, S., Yu, B., Chen, Y.-M., Wang, W., Song, Z.-G., Hu, Y., Tao, Z. W., Tian, J. H., Pei, Y. Y., Yuan, M. L., Zhang, Y. L., Dai, F. H., Liu, Y., Wang, Q. M., Zheng, J. J., Xu, L., Holmes, E. C., \& Zhang, Y.-Z. (2020). A new coronavirus associated with human respiratory disease in China. Nature, 579(7798), 265-269. https:// doi.org/10.1038/s41586-020-2008-3.

2. Metcalfe, S. M. (2020). Mesenchymal stem cells and management of COVID-19 pneumonia. Medicine in Drug Discovery, 100019. https://doi.org/10.1016/j.medidd.2020.100019. 
3. Nidovirales. (2012). In Virus taxonomy (pp. 784-794). Elsevier. https://doi.org/10.1016/b978-0-12-384684-6.00066-5.

4. Wong, A. C. P., Li, X., Lau, S. K. P., \& Woo, P. C. Y. (2019, February 1). Global epidemiology of bat coronaviruses. Viruses. MDPI AG. https://doi.org/10.3390/v11020174.

5. Woo, P. C. Y., Wang, M., Lau, S. K. P., Xu, H., Poon, R. W. S., Guo, R., ... Yuen, K. -y. (2007). Comparative analysis of twelve genomes of three novel group $2 \mathrm{c}$ and group $2 \mathrm{~d}$ coronaviruses reveals unique group and subgroup features. Journal of Virology, 81(4), 1574-1585. https://doi.org/10.1128/jvi.02182-06.

6. Zhou, P., Yang, X.-L., Wang, X.-G., Hu, B., Zhang, L., Zhang, W., Si, H. R., Zhu, Y., Li, B., Huang, C. L., Chen, H. D., Chen, J., Luo, Y., Guo, H., Jiang, R. D., Liu, M. Q., Chen, Y., Shen, X. R., Wang, X., Zheng, X. S., Zhao, K., Chen, Q. J., Deng, F., Liu, L. L., Yan, B., Zhan, F. X., Wang, Y. Y., Xiao, G. F., \& Shi, Z.-L. (2020). A pneumonia outbreak associated with a new coronavirus of probable bat origin. Nature, 579(7798), 270-273. https://doi.org/10.1038/ s41586-020-2012-7.

7. Zhu, N., Zhang, D., Wang, W., Li, X., Yang, B., Song, J., et al. (2020). A novel coronavirus from patients with pneumonia in China, 2019. New England Journal of Medicine. https://doi.org/ 10.1056/nejmoa2001017.

8. Park, W. B., Kwon, N. J., Choi, S. J., Kang, C. K., Choe, P. G., Kim, J. Y., ... Oh, M. D. (2020). Virus isolation from the first patient with SARS-CoV-2 in Korea. J Korean Med Sci, 35(7 PG-84-84), e84 e84. https://doi.org/10.3346/jkms.2020.35.e84.

9. Govorkova, E. A., Murti, G., Meignier, B., de Taisne, C., \& Webster, R. G. (1996). African green monkey kidney (Vero) cells provide an alternative host cell system for influenza A and B viruses. Journal of Virology, 70(8), 5519-5524. https://doi.org/10.1128/ jvi.70.8.5519-5524.1996.

10. Hoffmann, M., Kleine-Weber, H., Krüger, N., Müller, M., Drosten, C., \& Pöhlmann, S. (2020). The novel coronavirus 2019 (2019$\mathrm{nCoV}$ ) uses the SARS-coronavirus receptor ACE2 and the cellular protease TMPRSS2 for entry into target cells. bioRxiv, 2020.01.31.929042. https://doi.org/10.1101/2020.01.31.929042.

11. Li, F., Li, W., Farzan, M., \& Harrison, S. C. (2005). Structural biology: Structure of SARS coronavirus spike receptor-binding domain complexed with receptor. Science, 309(5742), 1864-1868. https://doi.org/10.1126/science.1116480.

12. WHO. (2020). WHO Director-General's opening remarks at the media briefing on COVID-19 - 11 March 2020. Retrieved March 14, 2020, from https://www.who.int/dg/speeches/detail/ who-director-general-s-opening-remarks-at-the-media-briefing-oncovid-19\%2D\%2D-11-march-2020.

13. Ji, Y., Ma, Z., Peppelenbosch, M. P., \& Pan, Q. (2020). Potential association between COVID-19 mortality and health-care resource availability. The Lancet Global Health, $O(\mathrm{C}), 30068$. https://doi.org/ 10.1016/S2214-109X(20)30068-1.

14. Baud, D., Qi, X., Nielsen-Saines, K., Musso, D., Pomar, L., \& Favre, G. (2020). Real estimates of mortality following COVID19 infection. The Lancet Infectious Diseases, O(0). https://doi.org/ 10.1016/S1473-3099(20)30195-X.

15. (January 2020). Backer, J. A., Klinkenberg, D., \& Wallinga, J. (2020). Incubation period of 2019 novel coronavirus (2019$\mathrm{nCoV}$ ) infections among travellers from Wuhan, China, 20-28. Euro surveillance : bulletin Europeen sur les maladies transmissibles $=$ European communicable disease bulletin, 25(5). https://doi. org/10.2807/1560-7917.ES.2020.25.5.2000062.

16. WHO. (2020). Report of the WHO-China joint mission on coronavirus disease 2019 (COVID-19). https://www.who.int/docs/defaultsource/coronaviruse/who-china-joint-mission-on-covid-19-finalreport.pdf. Accessed Feb 2020.

17. Rothan, H. A., \& Byrareddy, S. N. (2020, February 26). The epidemiology and pathogenesis of coronavirus disease (COVID-19) outbreak. Journal of Autoimmunity. Academic Press. https://doi. org/10.1016/j.jaut.2020.102433.

18. Hoffmann, M., Kleine-Weber, H., Schroeder, S., Krüger, N., Herrler, T., Erichsen, S., ... Pöhlmann, S. (2020). SARS-CoV-2 cell entry depends on ACE2 and TMPRSS2 and is blocked by a clinically proven protease inhibitor. Cell https://doi.org/10.1016/j. cell.2020.02.052.

19. Hamming, I., Timens, W., Bulthuis, M. L. C., Lely, A. T., Navis, G. J., \& van Goor, H. (2004). Tissue distribution of ACE2 protein, the functional receptor for SARS coronavirus. A first step in understanding SARS pathogenesis. Journal of Pathology, 203(2), 631637. https://doi.org/10.1002/path.1570.

20. Baruah, V., \& Bose, S. (2020). Immunoinformatics-aided identification of T cell and B cell epitopes in the surface glycoprotein of 2019-nCoV. Journal of Medical Virology, 92(5), 495-500. https:// doi.org/10.1002/jmv.25698.

21. Bhattacharya, M., Sharma, A. R., Patra, P., Ghosh, P., Sharma, G., Patra, B. C., ... Chakraborty, C. (2020). Development of epitopebased peptide vaccine against novel coronavirus 2019 (SARSCOV-2): Immunoinformatics approach. Journal of Medical Virology, jmv.25736. https://doi.org/10.1002/jmv.25736.

22. Golchin, A., \& Farahany, T. Z. (2019). Biological products: Cellular therapy and FDA approved products. Stem Cell Reviews and Reports, 15(2), 1-10. https://doi.org/10.1007/s12015-018-9866-1.

23. Golchin, A., Farahany, T. Z., Khojasteh, A., Soleimanifar, F., \& Ardeshirylajimi, A. (2018). The clinical trials of Mesenchymal stem cell therapy in skin diseases: An update and concise review. Current Stem Cell Research \& Therapy, 14(1), 22-33. https://doi.org/10. 2174/1574888x13666180913123424.

24. Mehta, P., Mcauley, D. F., Brown, M., Sanchez, E., Tattersall, R. S., Manson, J. J., ... Collaboration, S. (2020). Correspondence COVID-19 : consider cytokine storm syndromes and. The Lancet, 6736(20), 19-20. https://doi.org/10.1016/S0140-6736(20)30628-0.

25. Leng, Z., Zhu, R., Hou, W., Feng, Y., Yang, Y., Han, Q., ... Zhao, R. C. (2020). Transplantation of ACE2- Mesenchymal stem cells improves the outcome of patients with COVID-19 pneumonia. Aging and Disease, 11(2), 216. https://doi.org/10.14336/ad.2020.0228.

26. Golchin, A., Shams, F., \& Karami, F. (2019). Advancing Mesenchymal stem cell therapy with CRISPR/Cas9 for clinical trial studies. In Advances in Experimental Medicine and Biology (pp. 1-12). Springer, New York, NY. https://doi.org/10.1007/ 55842019459

27. Novello, S., Debouche, A., Philippe, M., Naudet, F., \& Jeanne, S. (2020). Clinical application of mesenchymal stem cells in periodontal regeneration: A systematic review and meta-analysis. Journal of Periodontal Research, 55(1), 1-12. https://doi.org/10.1111/jre. 12684.

28. Zhao, K., \& Liu, Q. (2016, May 18). The clinical application of mesenchymal stromal cells in hematopoietic stem cell transplantation. Journal of Hematology and Oncology. BioMed Central Ltd. https://doi.org/10.1186/s13045-016-0276-z.

29. Chen, J., Hu, C., Chen, L., Tang, L., Zhu, Y., Xu, X., et al. (2020). Clinical study of Mesenchymal stem cell treatment for acute respiratory distress syndrome induced by Epidemic Influenza A (H7N9) infection: A hint for COVID-19 treatment. Engineering. https://doi. org/10.1016/j.eng.2020.02.006.

30. Bing Liang, Junhui Chen, Tao Li, Haiying Wu, Wenjie Yang, Yanjiao Li, J., Li, Congtao Yu, Fangang Nie, Zhaoxia Ma, Mingxi Yang, Panrong Nie, Y. G., \& Chuanyun Qian, M. H. (2020). Clinical remission of a critically ill COVID-19 patient treated by human umbilical cord. chinaXiv, https://doi.org/10.12074/ 202002.00084.

31. Prompetchara, E., Ketloy, C., \& Palaga, T. (2020). Immune responses in COVID-19 and potential vaccines: Lessons learned from SARS and MERS epidemic. Asian Pacific journal of allergy and 
immunology, 38(1), 1-9. https://doi.org/10.12932/AP-2002200772 .

32. Huang, C., Wang, Y., Li, X., Ren, L., Zhao, J., Hu, Y., ... Cao, B. (2020). Clinical features of patients infected with 2019 novel coronavirus in Wuhan, China. The Lancet, 395(10223), 497-506. https://doi.org/10.1016/S0140-6736(20)30183-5.

33. Weiss, A. R. R., \& Dahlke, M. H. (2019). Immunomodulation by Mesenchymal stem cells (MSCs): Mechanisms of action of living, apoptotic, and dead MSCs. Frontiers in Immunology, 10(JUN), 110. https://doi.org/10.3389/fimmu.2019.01191.

34. Glenn, J. D. (2014). Mesenchymal stem cells: Emerging mechanisms of immunomodulation and therapy. World Journal of Stem Cells, 6(5), 526-539. https://doi.org/10.4252/wjsc.v6.i5.526.

Publisher's Note Springer Nature remains neutral with regard to jurisdictional claims in published maps and institutional affiliations. 